\title{
ADDITIVE CATEGORIES AND A THEOREM OF W. G. LEAVITT ${ }^{1}$
}

\author{
BY A. L. S. CORNER
}

\author{
Communicated by Richard S. Pierce, July 24, 1968
}

The purpose of this note is to present a new proof of the following theorem of Leavitt.

THEOREM 1. Let $K$ be a commutative ring (with 1), and $q$ a positive integer. Then there exists a $K$-algebra $R$ such that, for positive integers $m, n$,

$$
R^{m} \cong R^{n} \text { if and only if } m \equiv n(\bmod q) .
$$

( $R^{m}$ denotes the free right $R$-module on $m$ generators.)

We remark at the outset that as in any case $K$ admits a homomorphism onto a field, an obvious change-of-ring argument shows that it is enough to prove that the theorem is valid in the case where $K$ is itself a field.

Three proofs have already been published, by Leavitt [5], by myself [2], and by Cohn [1]. All three take $R$ to be the $K$-algebra which is in a fairly obvious sense universal for the isomorphism $R^{q+1} \cong R^{1}$. To complete the proof, Leavitt shows that $R^{m} \cong R^{n}$ only if $m \equiv n(\bmod q)$ by means of a long and involved cancellation argument. As he is interested in rings rather than algebras he presents his proof for the case where $K$ is the field of 2 elements; but it should be remarked that with a little patience his argument can be adapted to the case where $K$ is an arbitrary field. The remaining two proofs, which are considerably shorter and simpler, avoid the cancellation argument with the help of suitable 'trace functions,' but they have the disadvantage that they fail completely unless the identity element of $K / q K$ has additive order exactly $q$.

The present proof has none of the disadvantages of the earlier proofs. Tackling the problem from an entirely new angle-it makes no use of the universal algebra already mentioned-it employs a quite trivial manipulation of infinite matrices to deduce the theorem from the familiar invariance of dimension of finite-dimensional vector spaces: the underlying idea is strikingly similar to that exploited by Hanf in his solution of an analogous problem for Boolean algebras [4].

1 This paper was written while the author was on sabbatical leave at Vanderbilt University, supported in part by NSF Grant GP 8725 . 
Although it would be possible to present the proof in the manner of a nineteenth-century text on Matrix Theory, it is notationally more convenient to obtain the theorem as a consequence of an embedding theorem for additive categories. For this we need a few ad hoc definitions. Let $K$ be a commutative ring, and let $\mathfrak{e}$ be a $K$-category, by which we mean an additive category such that, for objects $A, B$, $C$, the groups of morphisms $\mathbb{e}[A, B], \ldots$ are $K$-modules and multiplication $\mathfrak{e}[A, B] \times \mathfrak{e}[B, C] \rightarrow \mathfrak{e}[A, C]$ is bilinear; note that $\mathfrak{e}[A, A]$ is then always a $K$-algebra. An object $A(\neq 0)$ of $\mathfrak{C}$ will be called principal if the minimal sub- $K$-algebra $K \cdot 1_{A}$ of $\mathfrak{e}[A, A]$ is a principal-ideal domain (or field); a nonzero morphism of $\mathfrak{e}$ will be called principal if it belongs to $K \cdot 1_{A}$ for some principal object $A$.

THEOREM 2. Let $K$ be a commutative ring, and let $\mathrm{C}$ be a $K$-category with finite direct sums. Then $\mathfrak{e}$ may be embedded as a full subcategory of a $K$-category $\mathrm{C}^{*}$ with finite direct sums which contains, for each principal object $U$ of $\mathcal{e}$, an object $U^{*}$ such that

(i) $U^{*} \cong U \oplus U^{*} \cong U^{*} \oplus U^{*}$,

(ii) for objects $A, B$ of $\mathfrak{e}$,

$$
\left.A \oplus U^{*} \cong B \oplus U^{*} \quad \text { (in } \mathrm{e}^{*}\right)
$$

if and only if there exist nonnegative integers $r, s$ such that

$$
A \oplus U^{r} \cong B \oplus U^{\cdot} \quad \text { (in } \mathcal{C} \text { ). }
$$

To see that Theorem 1 follows from Theorem 2, let $K$ be an arbitrary field or, more generally, a principal-ideal domain, and take $\mathfrak{C}$ to be the $K$-category of all free $K$-modules of finite rank. Given a positive integer $q$, let $U=K^{q}, A=K^{1} \oplus U^{*}, R=\mathrm{e}^{*}[A, A]$; note that $U$ is indeed principal, because $K \cdot 1_{U}=K$. Now, in view of the standard matrix representations for homomorphisms between free right $R$-modules and for morphisms between direct sums of copies of the object $A$, it is clear that, for positive integers $m, n$, the following three conditions are equivalent:

(a) $R^{m} \cong R^{n}$;

(b) there exist an $m \times n$ matrix $X$ and an $n \times m$ matrix $Y$ over $R$ such that $X Y=1, Y X=1$;

(c) $A^{m} \cong A^{n}$.

But it follows at once from (i) that $A^{m} \cong K^{m} \oplus U^{*}, A^{n} \cong K^{n} \oplus U^{*}$. Therefore (ii) implies that (c) is equivalent to the existence of non-

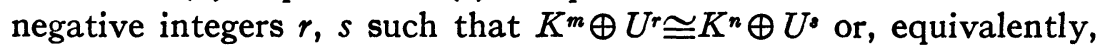
taking ranks, such that $m+r q=n+s q$. Visibly, a necessary and sufficient condition for this is that $m \equiv n(\bmod q)$, as required for the proof of Theorem 1. 
Proof of Theorem 2. For the objects of $\mathfrak{e}^{*}$ we take all families $A=\left(A_{i}\right)_{i \in I}$ of objects of $\mathbb{C}$ with the property that $A_{i}$ is principal for almost all $i \in I$. For objects $A=\left(A_{i}\right)_{i \in I}, B=\left(B_{j}\right)_{j \in J}$ of $\mathbb{e}^{*}$, a morphism $A \rightarrow B$ is to be a row-and-column-finite matrix $X=\left(x_{i j}\right)_{i \in I, j \in J}$ with $x_{i j} \in \mathfrak{e}\left[A_{i}, B_{j}\right]$, subject to the requirement that all but a finite number of the nonzero entries $x_{i j}$ be principal. A straightforward verification shows that $\mathrm{e}^{*}$ is a $K$-category under the usual rules for operating with matrices, the identity morphism $1_{A}$ of $A$ being, of course, the matrix with the identity morphisms of the $A_{i}$ in the appropriate positions along the diagonal and zeros elsewhere. Clearly $\mathfrak{C}$ may be identified with the full subcategory of $\mathrm{e}^{*}$ whose objects are indexed by the one-element set $\{0\}$. It is equally clear that two objects of $e^{*}$ which differ only in their indexing sets are isomorphic. Therefore, in order to check that two objects $A=\left(A_{i}\right)_{i \in I}, B=\left(B_{j}\right)_{j \in J}$ of $\mathfrak{e}^{*}$ have a direct sum in $\mathrm{e}^{*}$, we may assume that $I \cap J=\varnothing$. Take $C=\left(C_{k}\right)_{k \in I \mathrm{~J} J}$, where $C_{i}=A_{i}(i \in I), C_{j}=B_{j}(j \in J)$, and let $X$ and $Y[P$ and $Q]$ be the matrices obtained from $1_{C}$ by retaining only the rows [columns] indexed by the elements of $I$ and $J$, respectively. Then $C$ is an object and $X, Y, P, Q$ are morphisms of $\mathrm{e}^{*}$; and since

$$
\left[\begin{array}{l}
X \\
Y
\end{array}\right]\left[\begin{array}{ll}
P & Q
\end{array}\right]=\left[\begin{array}{cc}
1_{A} & \\
& 1_{B}
\end{array}\right], \quad\left[\begin{array}{ll}
P & Q
\end{array}\right]\left[\begin{array}{l}
X \\
Y
\end{array}\right]=1_{C}
$$

we have proved that $C$ is the $\operatorname{direct}$ sum of $A$ and $B$ in $\mathrm{e}^{*}$ (see Mitchell [6, Proposition I.18.1]).

Given a principal object $U$ of $\mathfrak{e}$, take $U^{*}=\left(U_{1}, U_{2}, \cdots\right)$, where $U_{i}=U(i=1,2, \cdots)$. It follows at once from what we have just proved that $U^{*}$ satisfies (i) and the 'if' part of (ii) in the statement of Theorem 2. For the 'only if' part of (ii), let $A, B$ be objects of $\mathfrak{e}$ such that

$$
\bar{A}=\left(A_{0}, A_{1}, \cdots\right)=A \oplus U^{*} \cong B \oplus U^{*}=\left(B_{0}, B_{1}, \cdots\right)=\bar{B},
$$

where $A_{0}=A, B_{0}=B, A_{i}=B_{i}=U(i=1,2, \cdots)$, and choose inverse isomorphisms $X: \bar{A} \rightarrow \bar{B}, Y: \bar{B} \rightarrow \bar{A}$, so that

$$
X Y=1_{\bar{A}}, \quad Y X=1_{\bar{B}} .
$$

By hypothesis, only a finite number of the nonzero entries of $X$ and $Y$ fail to lie in $K \cdot 1_{U}$; so we may choose a positive integer $l$ such that these exceptional entries all lie in the first $l$ columns of $X$ and the first $l$ rows of $Y$. Since $X$ and $Y$ are row-and-column-finite, we may further choose positive integers $m, n$ such that the nonzero entries of the first $l$ columns of $X$ [rows of $Y$ ] lie in the first $m$ rows of $X$ [columns of $Y$ ], and the nonzero entries in the first $m$ rows of $X$ 
[columns of $Y$ ] lie in the first $l+n$ columns of $X$ [rows of $Y$ ]. In this way we obtain block decompositions

$$
\begin{aligned}
& X=\left[\begin{array}{ccc}
X_{0} & X_{1} & 0 \\
0 & X_{2} & X_{3}
\end{array}\right]_{(\infty)}^{(m)}, \quad Y=\left[\begin{array}{ll}
Y_{0} & 0 \\
Y_{1} & Y_{2} \\
0 & Y_{3}
\end{array}\right]_{(\infty)}^{(l)} \begin{array}{l}
(n) \\
(l)
\end{array} \\
& \text { (l) }(n)(\infty) \\
& \text { (m) }(\infty)
\end{aligned}
$$

where the bordering parentheses indicate the numbers of rows and columns in the blocks. From (1) and (2) we may read off the equations

$$
\begin{aligned}
Y_{1} X_{1}+Y_{2} X_{2} & =1, \\
X_{2} Y_{1}=0, \quad X_{1} Y_{2} & =0,
\end{aligned}
$$

from which it follows easily that

$$
\begin{array}{ll}
X_{1} \cdot Y_{1} X_{1}=X_{1}, & Y_{1} X_{1} \cdot Y_{1}=Y_{1}, \\
X_{2} \cdot Y_{1} X_{1}=0, & Y_{1} X_{1} \cdot Y_{2}=0 .
\end{array}
$$

By (3), the $n \times n$ matrix $Y_{1} X_{1}$ is clearly idempotent, and our choice of $l$ guarantees that its entries all lie in the principal-ideal domain $K \cdot 1_{U}$; so there exists an invertible $n \times n$ matrix $S$ over $K \cdot 1_{U}$ such that, for some nonnegative integer $r \leq n$,

$$
S^{-1} Y_{1} \cdot X_{1} S=\left[\begin{array}{ll}
1 & \\
& 0
\end{array}\right]_{(n-r)}^{(r)} .
$$

Introducing the automorphism

$$
T=\left[\begin{array}{lll}
1 & & \\
& S & \\
& & 1
\end{array}\right]_{(\infty)}^{(l)}\left(\begin{array}{l}
(n) \\
\end{array}\right.
$$

of $\bar{B}$, we now replace $X, Y$ by the inverse isomorphisms $X T, T^{-1} Y$. The effect of this is to replace $X_{1}, X_{2}, Y_{1}, Y_{2}$ by $X_{1} S, X_{2} S, S^{-1} Y_{1}$, $S^{-1} Y_{2}$, respectively, and to leave the remaining blocks in the decompositions (2) unchanged. With the new $X$ and $Y$, (5) takes on the simpler form

$$
Y_{1} X_{1}=\left[\begin{array}{ll}
1 & \\
& 0
\end{array}\right]_{(n-r)}^{(r)} .
$$

Substitution from (6) in (3) and (4) reveals that the last $n-r$ columns of $X_{1}$ [rows of $Y_{1}$ ] and the first $r$ columns of $X_{2}$ [rows of $Y_{2}$ ] all vanish, so that by decomposing the central blocks of (2) in the obvious way we obtain finer block decompositions of the form 


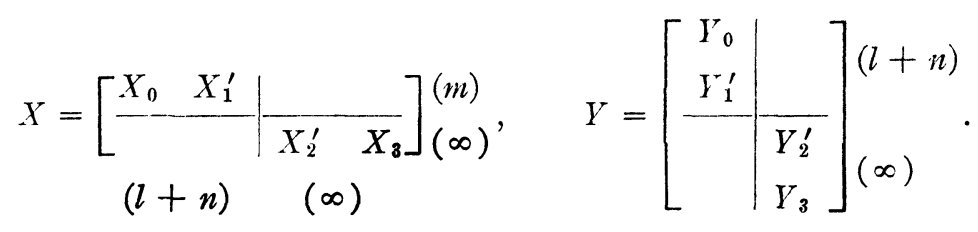

$$
\begin{aligned}
& \text { (m) }(\infty)
\end{aligned}
$$

But then it follows from (1) that the submatrices

$$
\left[\begin{array}{ll}
X_{0} X_{1}^{\prime}
\end{array}\right] \text { and }\left[\begin{array}{c}
Y_{0} \\
Y_{1}^{\prime}
\end{array}\right]
$$

are inverse isomorphisms between $\left(A_{0}, \cdots, A_{m-1}\right)=A \oplus U^{m-1}$ and $\left(B_{0}, \cdots, B_{l+n-1}\right)=B \oplus U^{l+n-1}$. The proof of Theorem 2 is complete.

We remark in conclusion that it is not difficult to extend the foregoing argument and show that a necessary and sufficient condition for two objects $A=\left(A_{i}\right)_{i \in I}, B=\left(B_{j}\right)_{j \in J}$ of $\mathfrak{C}^{*}$ to be isomorphic is that there exist finite subsets $I_{0} \subseteq I, J_{0} \subseteq J$, and a bijection $f: I \backslash I_{0} \rightarrow J \backslash J_{0}$ such that $\bigoplus_{i \in I_{0}} A_{i} \cong \oplus_{j \in J_{0}} B_{j}$ (in e) and $A_{i}=B_{f(i)}\left(i \in I \backslash I_{0}\right)$; we leave the details of the proof to the reader.

For an application of Theorem 1 to primary abelian groups, see [3].

\section{REFERENCES}

1. P. M. Cohn, Some remarks on the invariant basis property, Topology 5 (1966), 215-228.

2. A. L. S. Corner, On a conjecture of Pierce concerning direct decompositions of abelian groups, Proc Colloq. Abelian Groups (Tihany, 1963), pp. 43-48, Akadémiai Kiad6, Budapest, 1964.

3. - On endomorphism rings of primary abelian groups, (to appear).

4. William Hanf, On some fundamental problems concerning isomorphisms of Boolean algebras, Math. Scand. 5 (1957), 205-217.

5. W. G. Leavitt, The module type of a ring, Trans. Amer. Math. Soc. 103 (1962). 113-130.

6. Barry Mitchell, Theory of categories, Academic Press, New York, 1955.

Worcester College, OXford, England 